\title{
Influence of Grain Properties on Macro Mechanical Behaviors of Granular Media by DEM
}

\author{
DEM を用いた粒状材料の力学特性に及ぼす粒子特性の影響
}

\author{
Kenichi Maeda* and Hiroki Hirabayashi** \\ 前田健一 - 平林大輝 \\ * Dr. of Eng. Assoc. Prof. Dept. of Civil Eng. Nagoya Institute of Technology (Gokiso-cho, Showa-ku, Nagoya 466-8555) \\ ** M. of Eng. Grad. School of Civil Eng. Nagoya Institute of Technology (Gokiso-cho, Showa-ku, Nagoya 466-8555)
}

\begin{abstract}
Mechanical behaviors of granular media are controlled by grain properties and microstructure. The primary properties of granular media are denoted by grain shape, grain size distribution, and stiffness and the resistant friction angle between grains. Microstructures are formed at the connection paths of contact points between grains. In this paper, the deformation of granular material with different grain properties under different stress-levels and densities were simulated by DEM in two dimensions. On the basis of analysis results, we investigated the changes in fabric. We reveal the evolution rule of stress-induced anisotropy and its limitation and the existence of a critical state of fabric.
\end{abstract}

Key Words: fabric, friction, grain shape, DEM

\section{Introduction}

Granular material shows non-linear deformation behavior that is dependent on density and stress history. Moreover, even if they are under the same conditions, the mechanical properties of soils with different grain sizes and grain shapes should be intrinsically different.

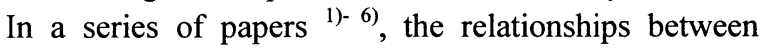
physical and mechanical properties with variations in confining stress and relative density were investigated extensively for granular samples with different primary properties (e.g. density, hardness, grain shape and grain size).

From the test results, it was revealed that the primary and physical properties of sand significantly influence its mechanical behavior and some reliable mutual relationships among them were identified. An index, void ratio extent $\left(e_{m a x}-e_{\text {min }}\right)$ which increases with the increasing angularity of the grain shape or with decreasing grain size, was selected as a promising parameter for evaluating the ductile deformability, the degree of confining stress and relative density dependencies. It was also indicated that grain crushability, which increases with angularity and grain size, also induces further reduction of shear strength with increasing confining stress. It can be said that grain shape brings a variation of contact conditions to a contact such as an unstable contact condition against rolling and a stable contact condition due to interlocking contact. Therefore, these effects of grain shape should be taken into account when simulating the ductile and compressive deformation behaviors of soils by Distinct Element Method (DEM). We employed non-circular particle elements in this paper.

The macroscopic behaviors of granular materials are controlled by the evolution and the collapse of microstructures. Stress-induced anisotropy in granular materials under shear stress has been observed in model tests ${ }^{7,8)}$. The author's research group have also been investigating changes of fabric by DEM ${ }^{9-12)}$. The evolution rule of anisotropy and useful critical relationships for fabric were revealed. Zhang and Thornton (2005) ${ }^{13)}$ also mentioned the existence of a critical value of fabric. In this paper, the evolution rule of geometric fabric indices such as fabric tensor and the coordination number were discussed.

\section{Numerical Simulation Procedure}

\subsection{DEM using non-circular particles}

The deformation behavior of granular materials was analyzed by the DEM ${ }^{14)}$. In this calculation, convenient contact elements between rigid disks (e.g., springs, dash-pots, sliders and non-extensional elements) were employed and a PFC2D code was utilized. Circular (Fig.1a) and non-circular (Figs.lb-e) particle elements were considered. For example, the non-circular particles shown in Fig.lb were prepared by connecting three 
circular particles of the same radii; these particles were clumped. The names of circular and non-circular particles were denoted by index "cl" added by number of connecting particles. Simulations were performed for a material composed entirely of circular particles, and then all circular particles were replaced by non-circular particles. The parameters for particle elements used are described in Table 1.

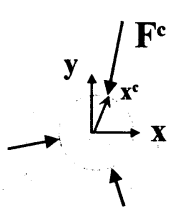

(a)

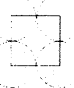

(c)

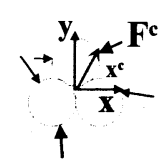

(b)

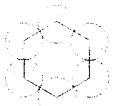

(d)

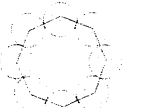

(e)
Fig.1 Particles types used in the DEM: (a) c101 (circle), (b) c103 (triangle), (c) c104 (quadrate), (d) c106 (hexagon), (e) c108 (octagon), where a broken line is circumscribing circle.

Table 1 Analysis parameters of particle elements in DEM.

\begin{tabular}{cc}
\hline Parameters & Particle \\
\hline Density $\left(\mathrm{kg} / \mathrm{m}^{3}\right)$ & 2700 \\
Diameters $(\mathrm{mm})$ & 5 to 10 \\
Normal Spring Coefficient $k_{n}(\mathrm{~N} / \mathrm{m} / \mathrm{m})$ & $5 \times 10^{8}$ \\
Tangential Spring Coeff. $k_{s}(\mathrm{~N} / \mathrm{m} / \mathrm{m})$ & $5 \times 10^{7}$ \\
Normal Damping & Critical \\
Tangential Damping & Critical \\
Resistant Friction Angle $\phi_{\mu}(\mathrm{deg})$. & $\tan \phi_{\mu}=0.25^{*}$ \\
\hline
\end{tabular}

*: This is the reference value, but results with $\tan \phi_{\mu}$ of 0 -1000 will appear in this paper.

\subsection{Preparation of samples and biaxial test}

The analysis involved approximately 4000 particles and four wall boundary elements. The external stress and strain were controlled by the movement of walls. The tests were simulated under the zero gravity conditions to investigate the change in fabric due only to the change in macro stresses.

Specimens were prepared as follows. First, circular particles with friction coefficient $\tan \phi_{\mu 0}$ were generated such that the porosity was equal to $n_{0}$ in the region enclosed by the boundary walls. At $n_{0}=0.40$, there were no contact points and initial packing was loose. At $n_{0}=0.10$, initial packing was dense. Each circular particle was then replaced by a 'cl' particle with $\tan \phi_{\mu 0}$. The initial stress was regulated to the required value of $\sigma_{m 0}=k_{\mathrm{n}} \times 10^{-4}(50 \mathrm{kPa})$. Finally, the friction coefficient was changed instantaneously from $\tan \phi_{\mu 0}$ to $\tan \phi_{\mu}$ and the initial void ratio $e_{0}$ was obtained under $\sigma_{m 0}$.

Figures $2 \mathrm{a}$ and $\mathrm{b}$ show the change in the void ratio $e_{0}$ with $\tan \phi_{\mu 0}$ for $n_{0}=0.40$ and 0.10 . The void ratio $e_{0}$ increases with $\tan \phi_{\mu 0}$ and initial porosity $n_{0}$. On the basis of these results, the initial density of the specimen can be regulated easily by adjusting $\tan \phi_{\mu 0}$ and $n_{0}$. For the most dense specimen, we set $\tan \phi_{\mu 0}=0.0$ and $n_{0}=0.10$, whereas for the most loose specimen, $\tan \phi_{\mu 0}=1.0$ and $n_{0}=0.40$. Therefore, minimum and maximum void ratios $e_{\max }$ and $e_{\min }$ could be obtained. The void ratios $e_{\max }$ and $e_{\min }$ are shown in Fig.3.
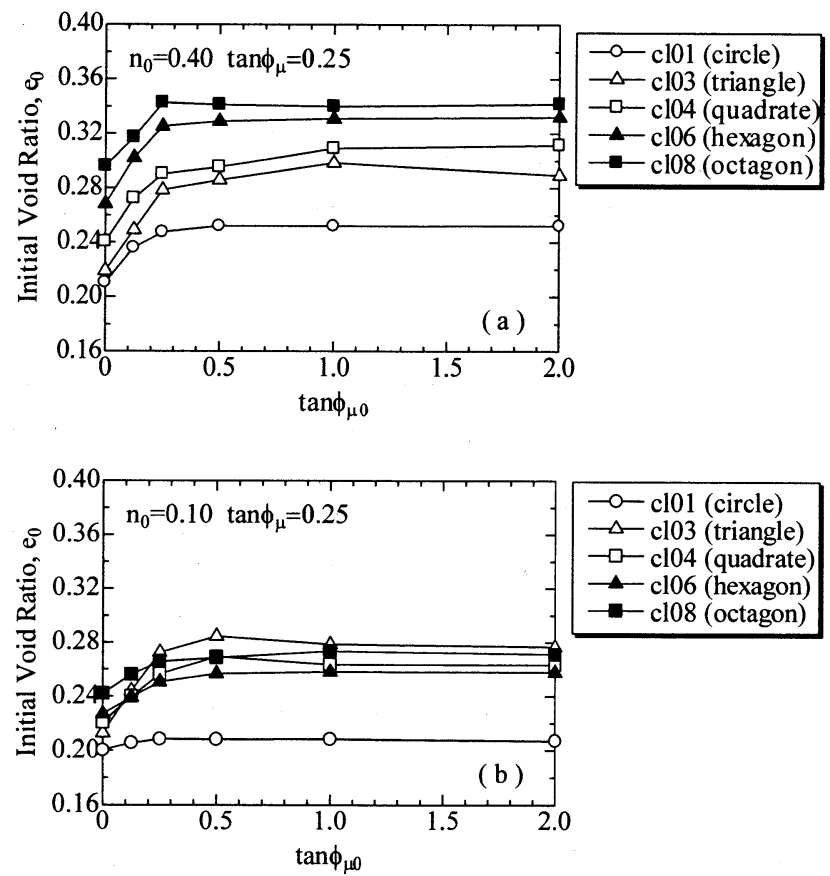

Fig. 2 Void ratio $e_{0}$ after conversion from initial resistant friction angle $\tan \phi_{\mu 0}$ to $\tan \phi_{\mu}(=0.25)$ : (a) initial porosity $n_{0}=0.40$, (b) $n_{0}=0.10$.

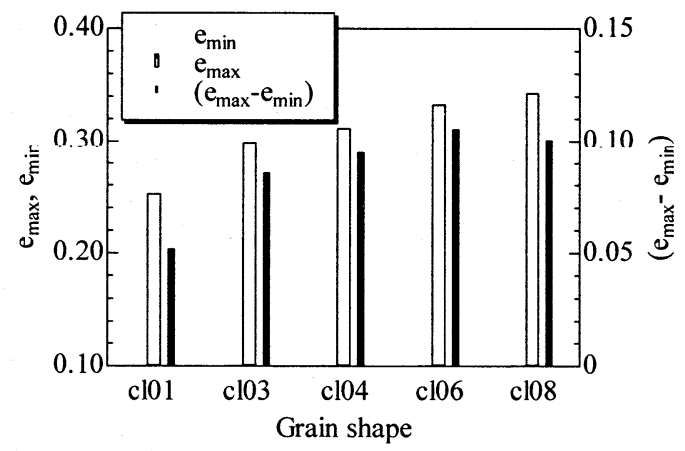

Fig. 3 Ratios $e_{\max }, e_{\min }$ and the extent of void ratio $\left(e_{\max }-\right.$ $\left.e_{\min }\right)$ and with a different grain shape obtained from Fig.2a and Fig.2b.

Stresses were then applied to the prescribed consolidation stress. Both stress and strain are represented by three mode components. The subscripts $p$ and $s$ denote pure shear and simple shear modes, respectively. And $\sigma_{m}$ is the mean normal stress and $\varepsilon_{v}$ is the volumetric strain in two dimensions. The maximum shear stress and strain are presented by $\tau_{m}$ and $\gamma_{m}$. Normal strains $\varepsilon_{x x}$ and $\varepsilon_{y y}$ in coordinate $\mathrm{x}-\mathrm{y}$ axes correspond with the directions of minor and major principal stresses $\sigma_{2}$ and $\sigma_{1}$, re- 
spectively.

\section{Numerical Simulation Results on Macro Behaviors}

\subsection{Grain Shape effect}

Figure 4 shows deformation-failure behaviors of samples with circular particles.

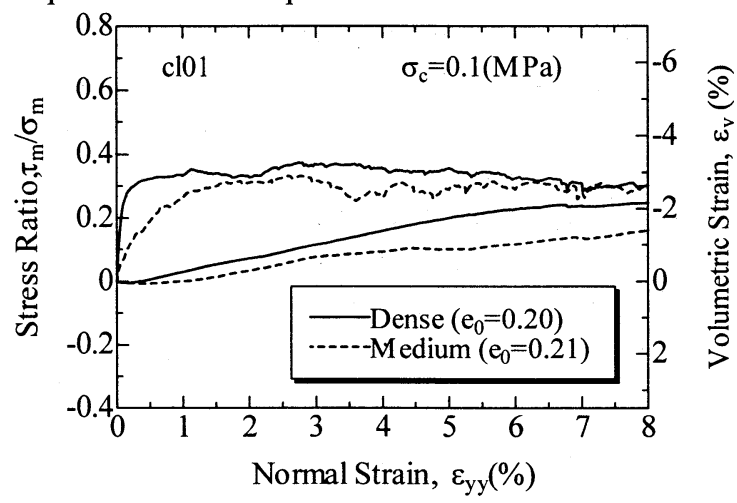

Fig. 4 Deformation-failure behaviors under constant confining stress, for circular particles (c101).

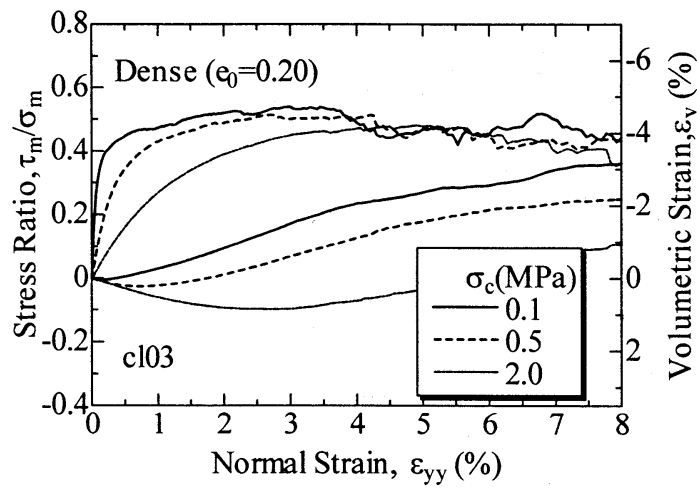

Fig. 5 Stress level dependence of deformation-failure behavior under constant confining stress, for densely packed non-circular particles (cl03).

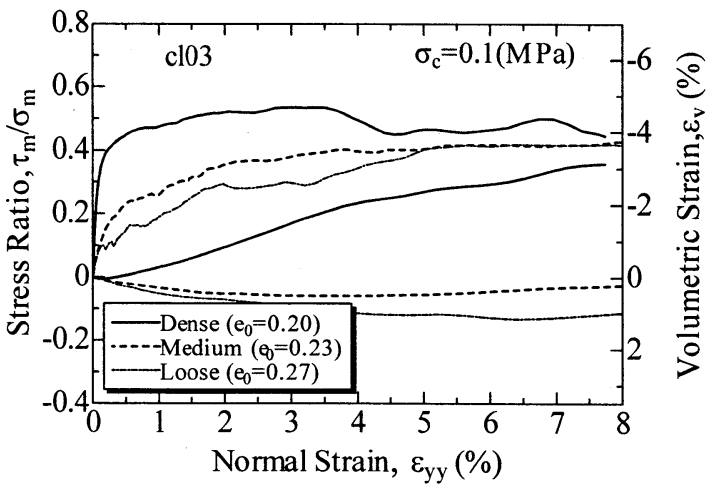

Fig. 6 Density dependence of deformation-failure behavior under constant confining stress for non-circular particles (cl03).
Figures 5 and 6 show the confining stress dependence and density dependence of deformation behaviors for samples of non-circular particles (c103), respectively. The strength and dilative strain increase with density. The maximum stress ratio and dilative strain decrease with increasing confining stress. The relationships between the void ratio and the mean normal stress under isotropic compression and shear also are presented in Fig. 7. As observed in experiments, the specimens with lower density and/or with higher confining stress exhibit a lower strength and higher contractive deformation. Non circular particle sample in Fig. 6 shows higher strength and larger contraction strain and failure strain. Moreover, the density dependency for non-circular sample is more clear than circular particle. This means that we can simulate the wide range of stiffness, contraction and strength by employing circular particles.

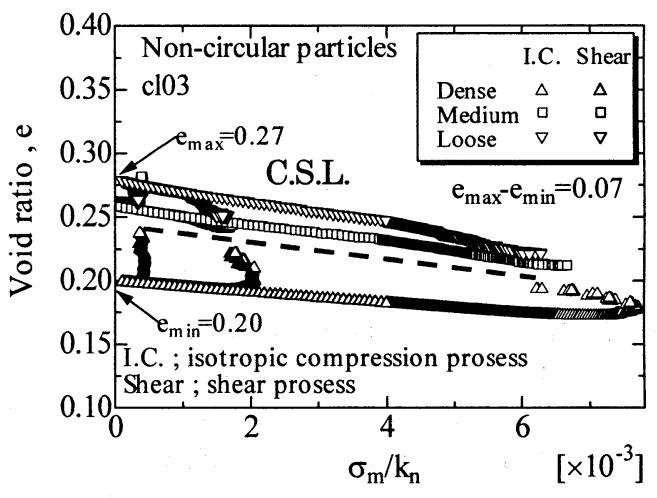

Fig. 7 Relationships between yhr void ratio and mean normal stress under isotopic compression (I.C.) and shear: c103.

As shown in Fig.7, there seems to be a critical state line (indicated by a broken line). However, in this case, a little difference between the critical states for dense and loose specimens could be found. This indicates that $e-\sigma_{m}$ relations must be different outside and inside the shear band since deformation localization tends to occur in dense specimens. A detailed discussions on mechanical behaviors of micro zones in specimens can be referred in later some papers ${ }^{15)}, 16$.

Figures $8 \mathrm{a}$ and $\mathrm{b}$ show the density dependence of deformation behavior under the constant volume condition in terms of the stress-strain curve and stress path, where $\sigma_{c 0}$ is the confining pressure at initial state of shearing. In the case of the lowest density $\left(e_{0}=0.24\right)$, it can be seen that brittle deformation occurs, causing fluid flow and reducing the effective mean normal stress remarkably. This resembles to static liquefaction. This fluidity reduces and the ductility increases with increasing density. These behaviors have also been observed in experiments ${ }^{17}$. The density and stress level dependencies even under constant volume can therefore be simulated well through the use of non-circular particles. 


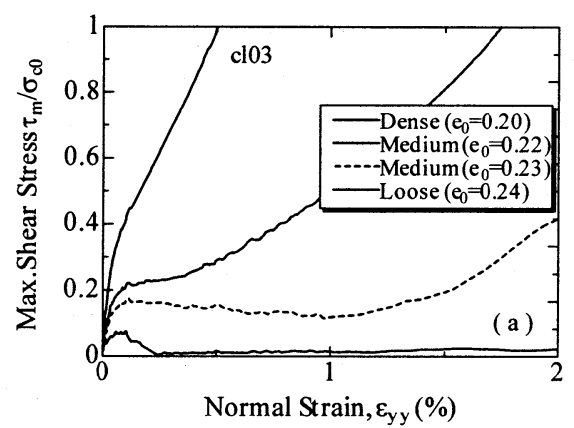

(a) stress ratio vs. strain

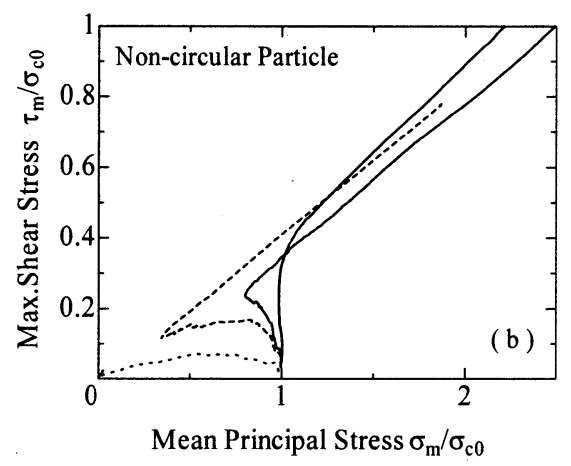

(b) stress path

Fig. 8 Density dependency of deformation-failure behavior under a constant volume condition, for $\mathrm{c} 103$.

Figure 9 shows stress-dilatancy behaviors (flow rule) in dense samples with different grain shapes in the pre-failure state. The figure also shows also Rowe's relationship ${ }^{18)}$ for $\phi_{\mu}$ of $25^{\circ}$. The data for non-circular particles diverge from Rowe's flow rule. These results may suggest that even if inter-particle friction coefficients are the same, granular materials with different grain shapes have different flow rules which link their strength to the volumetric response. The samples for non-circular particles show higher strength at $d \varepsilon_{y y} / d \varepsilon_{x x}=1$ and a steeper inclination of

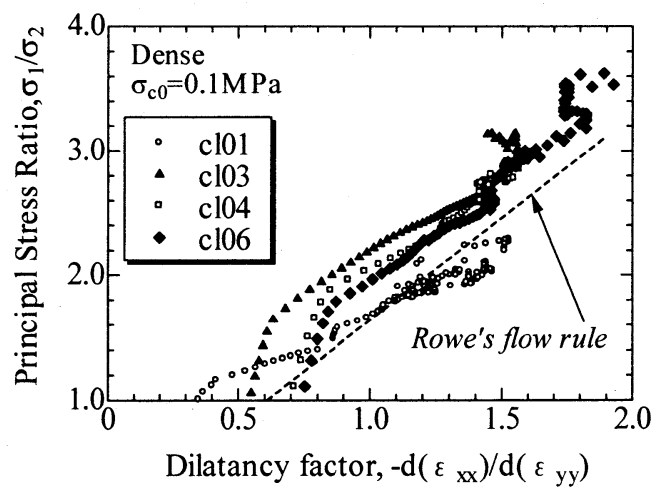

Fig. 9 Effect of grain shape on stress-dilatancy behaviors for cl01, cl03, cl04 and cl06; dense packing and $\sigma_{\mathrm{c}}$ $=0.1(\mathrm{MPa})$. plots than that for circular particle does. This implies that the interlocking between angular grains brings two effects on macro behaviors: increment of compressibility and an increment of strength in the dilation phase (it could be called ductility).

\subsection{Grain friction effect and grain rotation constraint effect}

In a distinct system such as granular materials, sliding and rotation among grains are quintessential micro phenomena. Friction restrains the micro sliding, and it is believed in general that inter-particle friction is the most important factor controlling macro strength. Otherwise, for grain rotation, some theories about the grain rolling resistance mechanism have been proposed ${ }^{19)}$.20). We must treat grain rolling resistance as a variable depending on contact conditions but not as a material parameter.

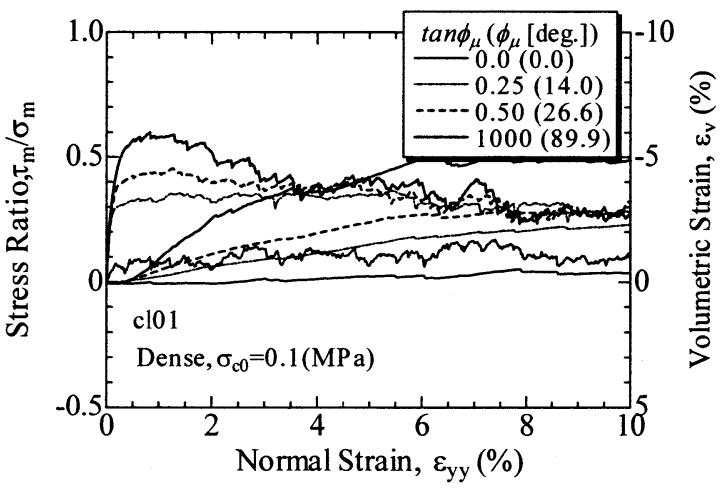

(a) for cl01(circular particle)

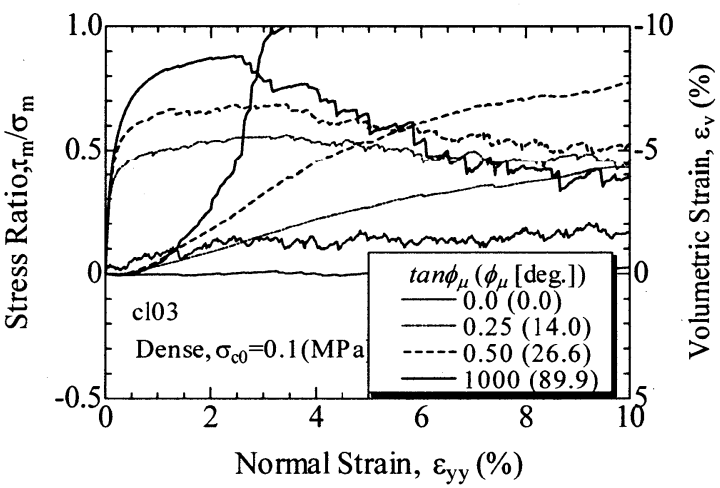

(b) for cl03(non-circular particle)

Fig. 10 Deformation-failure behaviors with a variation of $\tan \phi_{\mu}$; (a) for circular particles (c101); (b) for non-circular particles (c103).

Figures $10 \mathrm{a}$ and $10 \mathrm{~b}$ show the mechanical behaviors of specimens with different inter-particle friction coefficients for cl01 and cl03, respectively: In tests for each of grain shapes, void ratios of the samples are same at the initial state of shearing. Here, we use an extreme value of $\tan \phi_{\mu}=1000$ to highlight the effect of friction. The strength and the dilative tendency increase 
with inter-particle friction. In the case of higher $\tan \phi_{\mu}$, we can find clearly that remarkable strain-softening occurs. But the residual strengths seem to be almost the same for the same grain shape and the strength for $\mathrm{cl03}$ is higher than that for $\mathrm{cl01}$. This implies that residual strength at critical states is influenced by the strength of the fabric formed by axial contact force chains rather than inter-particle friction. Figure 11 shows stress-dilatancy behaviors for cl01 (corresponding to Fig.10 a). The data intend to shift downward according to the Rowe's flow rule, except for the case of $\tan \phi_{\mu}=0$. Rowe's flow rule was derived by focusing on the micro slip between particles. But macro deformation indeed seems to be induced by other micro processes.

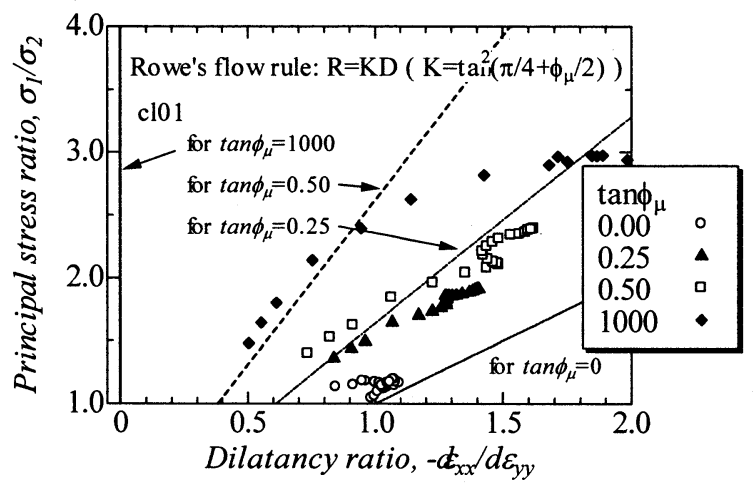

Fig. 11 Stress-dilatancy relations corresponding to Fig.10a.

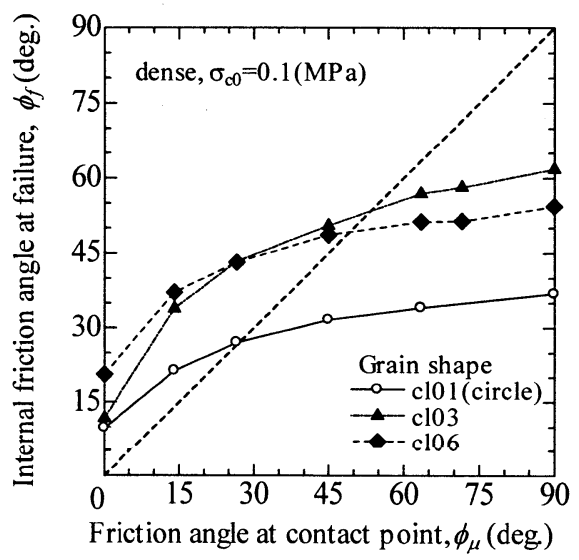

Fig. 12 Macro friction angle at failure $\phi_{f}$ and inter-particle friction angle $\phi_{\mu}$.

Moreover, Figure 12 shows the relationship between the macro internal friction angle at failure $\phi_{f}$ and the inter-particle friction angle $\phi_{\mu}$ for grain shapes c101, cl03 and cl06. In all cases, even if $\phi_{\mu}=0$, macro strength could be induced to about $\phi_{f}=10^{\circ}$ at least and the minimum value is dependent on the grain shape. This minimum strength is induced by only rearrangement and interlocking without friction. Then, in the range of $\phi_{\mu}<30^{\circ}$ for cl01 and $\phi_{\mu}<45^{\circ}$ for cl03 and cl06, $\phi_{f}$ is greater than $\phi_{\mu}$; this is widely accepted. However, in the other range of $\phi_{\mu}$ (higher value), $\phi_{f}$ is less than $\phi_{\mu}$; besides, $\phi_{f}$ converges to a limit value even at $\phi_{\mu}$ closed to $90^{\circ}$. Baar ${ }^{21)}$ also showed the same tendency and observed a detailed distribution of contact forces. The converged limit values for $\mathrm{cl} 03$ and cl06 at $\phi_{\mu}=90^{\circ}$ are higher than that for cl01. Although the values of $\phi_{\mu}$ for sands are less than about $30^{\circ}$, shown by Rowe (1962), these results for $\phi_{\mu}$ of higher values are interesting and suggestive in revealing the failure mechanism of granular media. The traditional relation that $\phi_{f}$ is summed up of $\phi_{\mu}$ and the dilatancy angle can be no longer upon and then this means that another effect (rolling resistance) is very significant on macro strength.

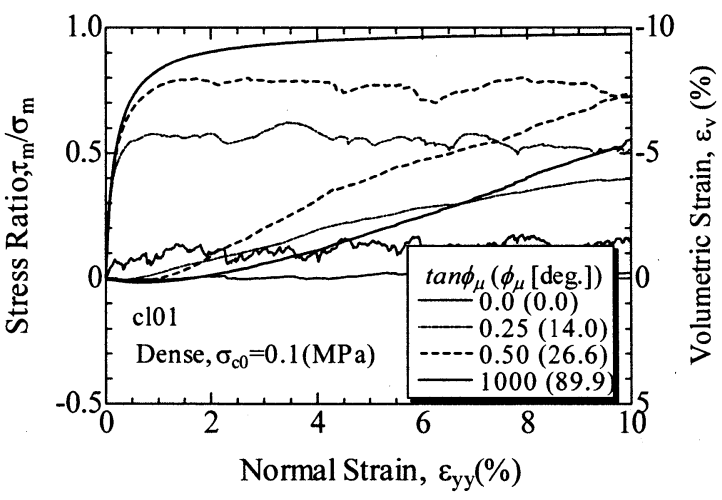

Fig. 13 Deformation-failure behaviors with a variation of $\tan \phi_{\mu}$ with constraint of particle rotation.

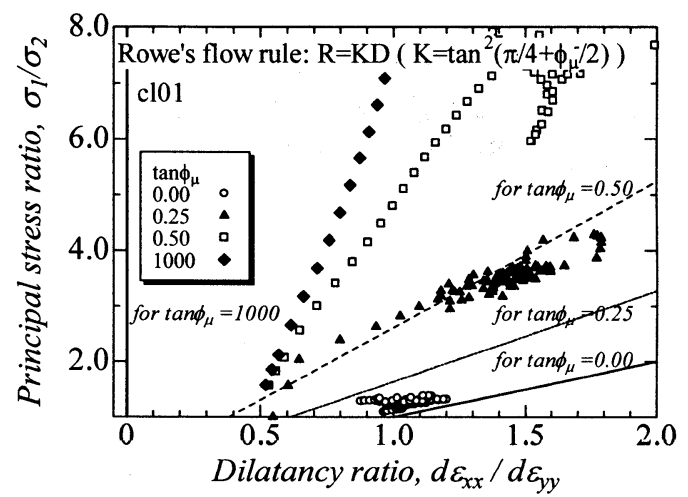

Fig. 14 Stress-dilatancy relations corresponding to Fig.13.

Furthermore, the behaviors of the circular particle assembly (cl01) with constraint of all particle rotation are plotted in Fig. 13 and referred in Fig. 10a. Figure 14 shows the stress-dilatancy plots. The stress-strain curves in this figures shift upward than that in Fig. 10a. In addition, in the flow rule, the plot in Fig.14 shifts more upward and the inclination is steeper than that in Fig.11. These effects, induced by particle rotation constraint are similar to grain shape effects in Fig.9.

The grain shape effect includes the effect of particle 
rota-tion constraint especially in the positive dilatation phase. For $\tan \phi_{\mu}=1000\left(\phi_{\mu}\right.$ closed to $\left.90^{\circ}\right)$, the strength is extremely high and the positive dilatancy shows up later than it does at $\tan \phi_{\mu}=0.25$ and 0.50 . The above tendencies indicate that macro deformation and failure of granular media is induced not only by inter-particle slip but also by particle rotation due to the buckling of the column structure of particles. Stress-induced anisotropy directed to the major principal stress, which is induced by the major loss of fabric in the direction of minor principal stress, causes the positive dilatancy ${ }^{1,8)}$. Therefore, for the case of constraint of particle rotation and $\tan \phi_{\mu}=1000$, the buckling of the structure is difficult to bring about, and then positive dilative deformation caused by loss of fabric is equally difficult to cause. Grain shape could mobilize interlocking (particle rotation resistant) which plays a role on the resistance of the occurrence of buckling of microstructures. This means that the rotation constraint must be effective reinforcement in geotechnics.

\section{Numerical Simulation Results on Micro Behaviors}

\subsection{Geometric fabric indices}

In this paper, coordination number and fabric tensor were focused on in order to assess the fabric change during macro deformation. Coordination number $N_{c}$ represents the averaged number of contacts per particle and indicates the overall stability of the fabric. And the major, $F_{l}$, and minor, $F_{2}$, principal values of fabric tensor are proposed by Satake ${ }^{22}$. They indicate the concentration intensity of the normal direction frequency of contact planes; the ratio $F_{l} / F_{2}$ means intensity of anisotropy where $F_{1}+F_{2} \equiv 1$ by its definition. Here, the directions of $F_{1}$ and $F_{2}$ correspond to the major and minor principal stress directions, respectively. Therefore, $N_{c} F_{1}$ and $N_{c} F_{2}$ mean averaged contact number in directions of the principal anisotropy $F_{l}$ and $F_{2}$; they indicate fabric intensity over principal directions.

\subsection{Evolution and Degradation of Fabric}

\section{(1) Coordination number}

Figures $15(\mathrm{a}, \mathrm{b})$ show relationships between $N_{c}$ which were counted for $\left|f_{c}\right|>0$ and $\sigma_{m}$, where $f_{c}$ is contact force at contact point. We can see that $N_{c}$ increases with $\sigma_{m}$ under isotropic compression, in other cases $N_{c}$ decreases and/or increases under shearing. It is no doubt that $N_{c}$ converges on a limit line described by a broken line. This indicates that there is a critical state line of $N_{c}$ in the same manner as $e$.

\section{(2) Stress induced anisotropy}

Figure 16 shows the relationships between $F_{1} / F_{2}$ and the principal stress ratio $\sigma_{1} / \sigma_{2}$ for all cases under a pre-failure state. In this figure, the non-sliding contacts were counted. At these contacts contact stiffness both in normal and tangential directions work. Despite of all cases with different grain properties, densities, stress levels and test conditions, $F_{l} / F_{2}$ can be ap- proximated by a unique function of only $\sigma_{1} / \sigma_{2}$ :

$$
F_{1}: F_{2}=\sqrt{\sigma_{1}}: \sqrt{\sigma_{2}}
$$

This unique evolution rule of stress-induced anisotropy is useful for developing a constitutive model.

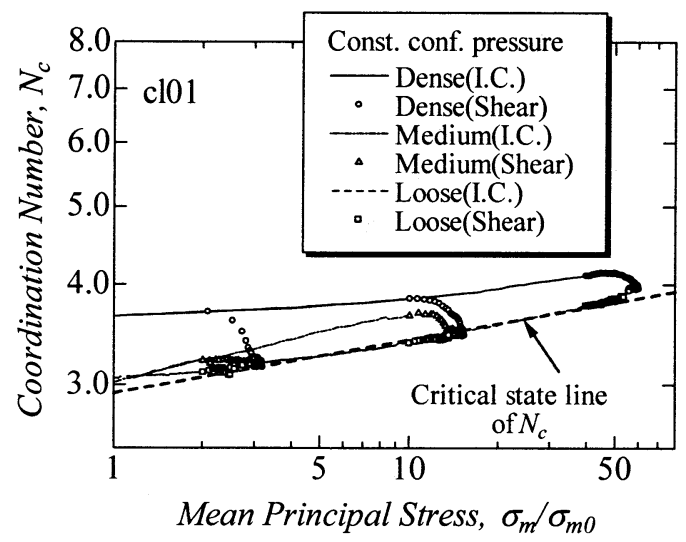

(a)

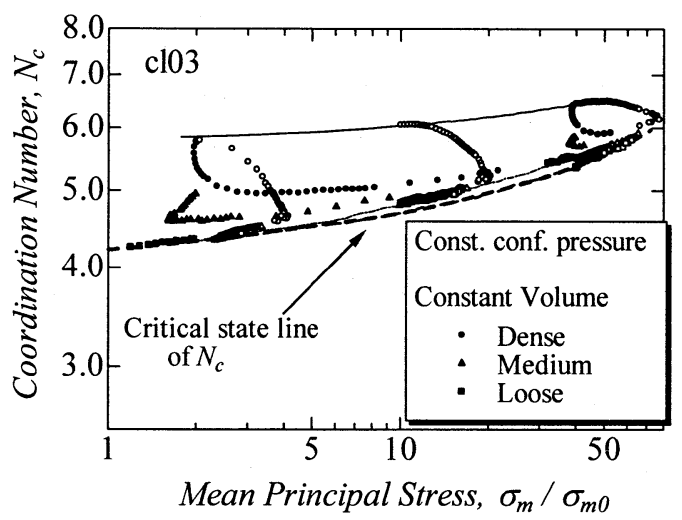

(b)

Fig. 15 Relationships between coordination number $N_{c}$ and mean normal stress under isotropic compression and shearing; (a) cl01; (b) cl03 under constant confining pressure and constant volume tests.

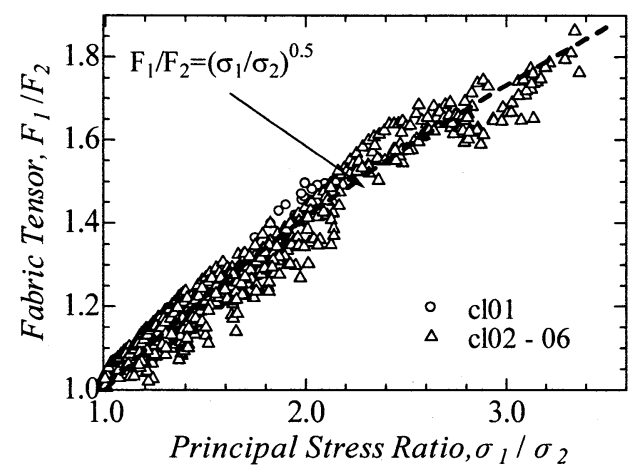

Fig. 16 Increase in principal fabric tensor ratio $F_{1} / F_{2}$ with principal stress ratio $\sigma_{1} / \sigma_{2}$ for the all cases. 

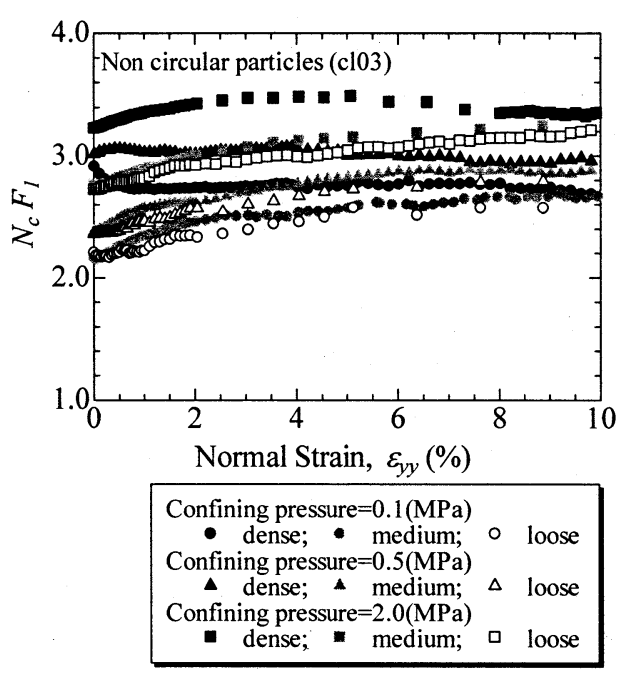

(a) $N_{c} F_{1}$

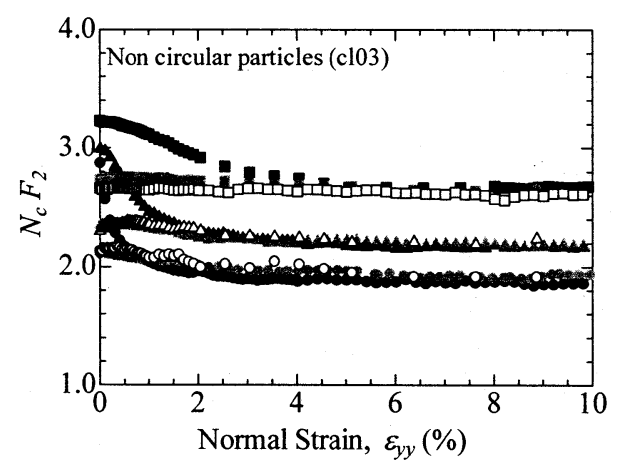

(b) $N_{c} F_{2}$

Fig. 17 Changes of fabric intensity indices $N_{c} F_{1}$ and $N_{c} F_{2}$ with macro deformation for cl03: (a) $N_{c} F_{1}$, (b) $N_{c} F_{2}$.

\section{(3) Fabric intensity}

As mentioned in 4.1, $N_{c} F_{1}$ and $N_{c} F_{2}$ indicate fabric intensity over principal directions. Here, the directions of $N_{c} F_{1}$ and $N_{c} F_{2}$ must be accordant to the direction of the major and minor principal stresses, respectively. Figure 17(a) shows that $N_{c} F_{l}$ does not change, rather it almost increases during shearing. On the other hand, $\mathrm{N}_{c} F_{2}$ decreases clearly with deformation as shown in Fig. 17(b). This means that fabric of granular material should deteriorate in the direction of minor principal stress under shearing and this loss of fabric causes stress-induced anisotropy. And the values of $N_{c} F_{1}$ and $N_{c} F_{2}$ of samples with initial confining stress, respectively, converge with the same values at the macro critical state even if initial densities are different. Figure $18(\mathrm{a}, \mathrm{b})$ show deviation of fabric indices (it could be called deviator fabric intensity) $N_{c}\left(F_{1}-F_{2}\right)$ with deformation for cl01

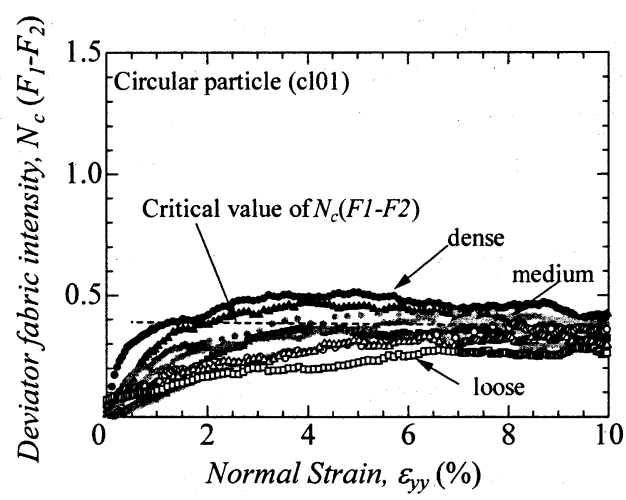

(a) for circular particle (cl01)

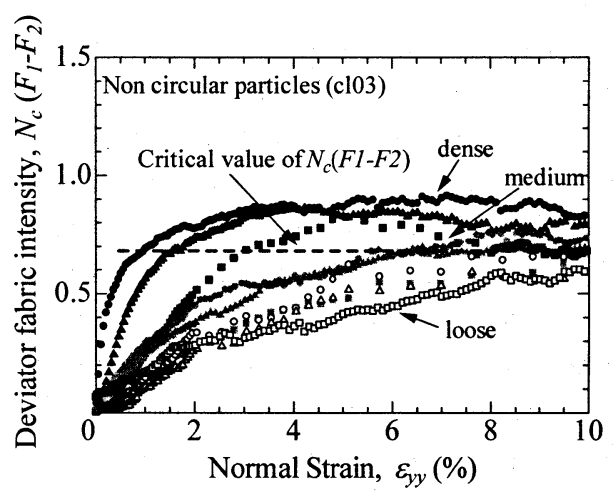

(b) for non-circular particle (cl03)

Fig. 18 Changes of deviator fabric intensity indices $N_{c} F_{1}$ and $N_{c} F_{2}$ with macro deformation: (a) cl01, (b) cl03.

and cl03, respectively. The shapes of the trajectories of plots are very similar to that of stress-strain curve. In the case of most dense sample it shows the peak and decrease after the peak; the peak value seems to depend on the density but be independent of confining pressure. Otherwise in the case of most loose sample it shows increase monotonically. Although the trajectories are depend on density and stress level, the sarmples with the same grain properties could show a unified critical value at macro critical state as shown by broken lines (it seem to be that more shear strain must be applied in simulations.). There, this means that each granular material has its own limit anisotropy.

\section{Conclusions}

Biaxial compression tests by two-dimensional DEM using not only circular particles but also non-circular particles were conducted. We can simulate ductile behaviors by employing non-circular particle with wide range of density. The relationships between macro and micro behaviors were investigated. 
Summary is as follows:

(1) It was shown that macro deformation and a failure of granular media were induced not only by inter-particle sliding but also by particle rotation due to the buckling of the particle structure. Grain shape creates interlocking and restrain the buckling.

(2) The coordination number $N_{c}$ which represented the stability of fabric was revealed to have a 'critical state' and to change with searching critical state; this corresponds to the relation $e-p-q$ in critical state soil mechanics.

(3) Stress-induced anisotropy denoted by the ratio of the principal value fabric tensor $F_{1} / F_{2}$ was confirmed to be approximated by the square root power relation given by Eq. (1). This relation is independent of grain properties and any test conditions.

(4) Fabric intensity was discussed. In the direction of major principal stress, the loss of fabric is almost non-exist. The loss of fabric in granular material is induced in the direction of minor principal stress. Deviator fabric intensity $N_{c}$ $\left(F_{1}-F_{2}\right)$ shows the similar trajectory to stress-strain relation. Each granular material has its own limit anisotropy.

(5) These evolution rules of fabric are very useful for modeling in geotechnics. Geomechanics is based on the critical state mechanics ${ }^{23)}$. The critical state is controlled by critical state of coordination number and anisotropy. More study is required to reveal the mechanism of these results.

\section{References}

1) Maeda, K., Miura, K. \& Toki, S., Mechanical properties of elliptic microstructure formed in granular materials, Soils and Foundations, Vol.35, No.2, pp.1-13, 1995.

2) Miura, K., Maeda, K. \& Toki, S., Method of measurement for the angle of repose of sands, Soils and Foundations, Vol. 37, No.2, pp.89-96, 1997.

3) Miura, K., Maeda, K., Furukawa, M. \& Toki, S. Physical characteristics of sands with different primary properties, Soils and Foundations, Vol.37, No.3, pp.53-64, 1997.

4) Miura, K., Maeda, K., Furukawa, M. \& Toki, S., Mechanical characteristics of sands with different primary properties, Soils and Foundations, Vol.38, No.4, pp.159-172, 1998.

5) Maeda, K. \& Miura, K. , Confining stress dependency of mechanical properties of sands, Soils and Foundations, Vol.39, No.1, 53-68, 1999.

6) Maeda, K. \& Miura, K., Relative density dependency of mechanical properties of sands, Soils and Foundations, Vol.39, No.1, pp.69-80, 1999.

7) Oda, M. Co-ordination number and its relation to shear strength of granular material, Soils and Foundations, Vol.17, No.2, pp.29-42, 1977.

8) Oda, M., Nemat-Nasser, S. \& Konishi, J., Stress-induced anisotropy in granular masses, Soils and Foundations, Vol.25, No.3, pp. 85-97, 1985.

9) Kuwabara, N. and Maeda, K., Processes of development and disappearance of micro-structure in granular materials with different grain properties by distinct element method analysis, Journal of Applied Mechanics, JSCE, Vol.3, 469-480, 2000.

10) Kuwabara, N. Ohno, R. and Maeda, K., Change in fabric of granular materials under different densities and stress-histories by DEM, Journal of Applied Mechanics, JSCE, Vol.5, 431-440, 2002.

11) Sakurai, T., Ohmura, A. \& Maeda, K., Influence of grain shape and fabric evolution on cyclic behavior of granular media, Proc. of anmual national conference of geotechnics, JGS, CD-ROM, 2005.

12) Ohmura, A., Sakurai, T. \& Maeda, K. , Observation on fabric evolution of granular media with different grain shape by DEM, Proc. of anmual national conference of geotechnics, JGS, CD-ROM, 2005.

13) Zhang, L. \& Thomton, C. , Characteristics of granular media at the 'critical state', Powder \& Grains 2005, Taylor \& Francis Group, London, pp.267-270, 2005.

14) Cundall, P. A., and O. D. L. Strack., A Discrete Model for Granular Assemblies, Geotechnique, Vol.29, No.1, pp.47-65, 1979.

15) Desrues, J. et al., Void ratio evolution inside shear bands in triaxial sand specimens studies by computed tomography, Geotechnique, Vol.46, No.3, pp.529-546, 1996.

16) Maeda K., Hara Y. and Ohno R. Interaction between piles with different skin roughness and granular ground by DEM. VII International Conference on Computational Plasticity (COMPLAS), Barcelona, CD-ROM, 2003.

17) Ishihara, K., Soil behavior in earthquake geotechnics. Oxford: Oxford Science Publications, 1996.

18) Rowe, P.W., The stress Dilatancy relation for static equilibrium of an assembly of particles in contact, Proc. $R$. Soc. London, Ser. A., 269, pp.500-527, 1962.

19) Oda, M. \& Iwashita, K. An Introduction mechanics of gramular materials, Rotterdam, BALKEMA, 1999.

20) Jiang, MJ, Yu, H-S and Harris, D., A novel discrete model for granular material incorporating rolling resistance., Computers and Geomechanics, Vol.32, pp.340-357, 2005.

21) Baars, S., Discrete element analysis of gramular materials, Doctoral Thesis, T. U. Delft, 1996.

22) Satake, M., Fabric tensor in granular materials. IU-TAM-Conference on Deformation and Failure of Gramular Materials, pp. 63-68, 1982.

23) WOOD, M. D., Soils behavior and critical state soil mechanics, Cambridge University Press, 1990.

(Received: April 13, 2006) 